\title{
Influence of long-term wearing of unstable shoes on compensatory control of posture: An electromyography-based analysis
}

\section{Andreia Sousa (MSc)}

Escola Superior da Tecnologia de Saúde do Porto, Instituto Politécnico do Porto

Área Científica de Fisioterapia,

Centro de Estudos de Movimento e Actividade Humana,

Rua Valente Perfeito, 322 - 4400-330 Vila Nova de Gaia, PORTUGAL

Faculdade de Engenharia, Universidade do Porto,

Rua Dr. Roberto Frias, s/n, 4200-465 Porto, PORTUGAL

E-mail: asp@estsp.ipp.pt/andreia.asps@gmail.com

Andreia Silva (Msc)

Faculdade de Engenharia, Universidade do Porto,

Rua Dr. Roberto Frias, s/n, 4200-465 Porto, PORTUGAL

E-mail: andreiasilva88@gmail.com

Rui Macedo (PhD)

Escola Superior da Tecnologia de Saúde do Porto, Instituto Politécnico do Porto

Área Científica de Fisioterapia

Centro de Estudos de Movimento e Actividade Humana

Rua Valente Perfeito, 322 - 4400-330 Vila Nova de Gaia, PORTUGAL

E-mail: rmacedo@estsp.ipp.pt

Rubim Santos (PhD)

Escola Superior da Tecnologia de Saúde do Porto, Instituto Politécnico do Porto

Área Científica de Física

Centro de Estudos de Movimento e Actividade Humana

Rua Valente Perfeito, 322 - 4400-330 Vila Nova de Gaia, PORTUGAL

E-mail: rss@estsp.ipp.pt

João Manuel R. S. Tavares (PhD)

Instituto de Engenharia Mecânica e Gestão Industrial,

Departamento de Engenharia Mecânica,

Faculdade de Engenharia, Universidade do Porto

Rua Dr. Roberto Frias, s/n, 4200-465 Porto, PORTUGAL

E-mail: tavares@fe.up.pt

(corresponding author)

Keywords: Postural control; unstable shoe wearing; adaptation; electromyography. 


\section{Abstract}

Purpose: This study investigated the influence of long-term wearing of unstable shoes (WUS) on compensatory postural adjustments (CPA) to an external perturbation. Methods: Participants were divided into two groups: one wore unstable shoes while the other wore conventional shoes for 8 weeks. The ground reaction force signal was used to calculate the anterior-posterior (AP) displacement of the centre of pressure (CoP) and the electromyographic signal of gastrocnemius medialis (GM), tibialis anterior (TA), rectus femoris (RF) and biceps femoris (BF) muscles was used to assess individual muscle activity, antagonist co-activation and reciprocal activation at the joint (TA/GM and $\mathrm{RF} /(\mathrm{BF}+\mathrm{GM})$ pairs) and muscle group levels (ventral (TA+RF)/dorsal $(\mathrm{GM}+\mathrm{BF})$ pair) within time intervals typical for CPA. The electromyographic signal was also used to assess muscle latency. The variables described were evaluated before and after the 8week period while wearing the unstable shoes and barefoot. Results: Long-term WUS led to: an increase of BF activity in both conditions (barefoot and wearing the unstable shoes); a decrease of GM activity; an increase of antagonist co-activation and a decrease of reciprocal activation level at the TA/GM and ventral/dorsal pairs in the unstable shoe condition. Additionally, WUS led to a decrease in CoP displacement. However, no differences were observed in muscle onset and offset. Conclusion: Results suggest that the prolonged use of unstable shoes leads to increased ankle and muscle groups' antagonist co-activation levels and higher performance by the postural control system. 


\section{Introduction}

Automatic postural responses to external perturbations are shaped by the sensory characteristics of the perturbation and by central nervous system (CNS) mechanisms related to expectations, attention, experience, environmental context and intention, as well as by pre-programmed muscle activation patterns called synergies [1]. Studies concerning postural perturbations have shown that postural response strategies become more efficient and effective in response to repeated exposure to a destabilising stimulus, as the automatic postural responses are gradually reduced in magnitude, and fewer or different muscles are recruited [2].

The underlying neural adaptations to balance training were shown to occur at different sites of the CNS [3]. Recent studies have demonstrated that training on unstable ground induces a decrease of corticospinal excitability and a suppression of the H-reflex as a result of modulation of presynaptic inhibition of Ia afferents [4, 5]. Exercises are commonly performed on ankle disks, balance boards, soft mats and unstable surfaces like 'wobble boards'. Recently, manufacturers have introduced specific shoes featuring unstable sole constructions to induce similar neuromuscular training stimuli. Previous research reported that these shoes improved reactive balance in children with development disabilities [6], improved static and dynamic balance in adults with osteoarthritis [7] and in middle-aged adults [8, 9], and also in young subjects in dynamic conditions like standing on a moveable platform [10]. Electromyography studies revealed changes in the ankle joint during quiet standing [8, 11], gait and running $[12,13]$. These are important findings since standing sway is highly correlated with ankle joint rotation, as muscles crossing this joint are able to provide the sensory information required to maintain upright standing [14, 15]. 
The main purpose of this study was to analyse the influence of long-term wearing of unstable shoes (WUS) on postural adjustments to an external anterior-posterior (AP) perturbation in terms of muscle latency and individual muscle activity and AP centre of pressure (CoP) displacement. Considering that the CNS controls muscles not only at an individual muscle level, but possibly also at a higher more functionally relevant level, such as at the joint level or at the muscle groups level [16-18], muscle synergies, expressed through reciprocal activation and co-activation indexes, were evaluated at these levels. The selection of these indexes was based on ideas expressed within the framework of the equilibrium-point hypothesis [19], particularly on the idea of two control variables, reciprocal and co-activation commands, describing the control of a single degree-of-freedom joint [20, 21].

We hypothesised a reduction in muscle activity and latency, changes in antagonist co-activation and reciprocal activation values, and a reduction in CoP displacement for long-term WUS. To the best of our knowledge, no previous study has analysed the influence of WUS on these variables.

\section{Methods}

\subsection{Subjects}

The study included 30 healthy female individuals distributed into two groups. The experimental group included 14 individuals (age $=34.6 \pm 7.7$ years, height $=1.59 \pm$ $0.06 \mathrm{~m}$, weight $=65.3 \pm 9.6 \mathrm{~kg}$; mean $\pm \mathrm{SD}$ ) and the control group included 16 individuals (age $=34.94 \pm 8.0$ years, height $=1.62 \pm 0.06 \mathrm{~m}$, weight $=61.1 \pm 6.3 \mathrm{~kg}$; mean \pm SD). Possible candidates were excluded if they presented a recent osteoarticular and musculotendinous injury or surgery of lower extremities, a background or signs of neurological dysfunction or medication that could affect motor performance and balance 
and individuals who had used unstable footwear (specifically Masai Barefoot Technology - MBT) prior to the study.

The study was conducted according to the ethical norms of the Institutions involved and conformed to the Declaration of Helsinki, with informed consent from all participants.

\subsection{Instrumentation}

The electromyographic signal (EMG) of the gastrocnemius medialis (GM), tibialis anterior (TA), rectus femoris (RF) and biceps femoris (BF) muscles was monitored using the MP 150 Workstation model from Biopac Systems, Inc. (USA), with silversilver chloride surface electrodes, TD150 model, with bipolar configuration and an interelectrode distance of $20 \mathrm{~mm}$ and a ground electrode. The rectus abdominis and erector spinae were not included as our findings related to short term changes (after one week of progressive adaptation to the shoes) showed that they did not play a significant role in reactive balance adjustments during perturbed stance.

The CoP displacement values were obtained using a force plate, model FP4060-10 from Bertec Corporation (U.S.A), connected to a Bertec AM 6300 amplifier, with default gains and a $1000 \mathrm{~Hz}$ sampling rate. The amplifier was connected to a Biopac 16 bit analogical-digital converter.

\subsection{Procedures}

\subsubsection{Skin preparation and electrode placement}

The subjects' dominant lower limb skin surfaces over the muscles midbelly were prepared to reduce the electrical resistance to less than $5000 \Omega$. The measurement electrodes were placed according to anatomical references and fixed with adhesive tape. 
Validation trials involving voluntary contractions were performed to verify the quality of the EMG signal.

\subsubsection{Data acquisition}

Each subject performed two tests: one standing barefoot and another with the unstable shoes, before and after an 8-week period. Subjects were instructed to stand relaxed, with feet comfortably spaced and arms at sides, and to look straight ahead to a target set 2 m away. Headphones were used to listen to music to mask any auditory cues and to distract the subjects from consciously modifying their motion. A horizontal cable was attached to a pelvic belt worn by the subjects while they kept their bodies essentially straight. A backward force of $5 \%$ of body weight, measured with an isometric dynamometer, was applied to the cable for a random period of 3 to 10 seconds and then the cable was released (time zero, $T_{0}$ ). Test instructions to the subject were: "Stand still but compensate for the force applied to the belt without moving your feet. I will let go at some point, but you will not know when. Do not move your feet, but keep your balance.”. The results obtained in a pilot study as to the inclination of the unstable shoes after applying the horizontal force demonstrated that the ankle dorsiflexion angle was not greater than $5^{\circ}$, which is not enough to produce changes in group Ia afferent feedback or in plantar and dorsiflexor muscle activity levels [22]. Each subject performed two randomised series, one for each condition under study, of three trials each separated by 1-minute rest intervals. As no noteworthy differences were verified between the first and the remainder of the trials of each series, the average values were used for analysis. Before data acquisition, all subjects were given time to become familiar with the test environment and were explained by a qualified instructor on how 
to use the unstable shoe, followed by approximately 10 minutes of walking, until the instructor felt they walked properly and were comfortable using the shoes [23].

The EMG signals were acquired at a sample rate of $1000 \mathrm{~Hz}$, pre-amplified at the electrode site, fed into a differential amplifier with an adjustable gain setting (12-500 Hz; Common Mode Rejection Ratio (CMRR) of $95 \mathrm{~dB}$ at $60 \mathrm{~Hz}$ and input impedance of $100 \mathrm{M} \Omega$ ), digitised and then stored in a computer for subsequent analysis based on the Acqknowledge software (Biopac Systems, Inc. USA). The gain range was set to 1000 .

The muscle latency was detected in a time window from -450 to $+200 \mathrm{~ms}$ in relation to $T_{0}$ [24] using a combination of computational algorithms and visual inspection [25]. The latency for a specific muscle was defined as the instant lasting for at least $50 \mathrm{~ms}$ when its EMG amplitude was higher (activation) or lower (inhibition) than the mean of its baseline value plus 1 (one) standard deviation (SD) [26], measured from -500 to $-450 \mathrm{~ms}$ [24]. The signal was previously smoothed using a sixth order elliptical low-pass software filter of $50 \mathrm{~Hz}$ [26].

To assess the level of muscle activity, signals were previously band-pass filtered between 20 and $450 \mathrm{~Hz}$ and integrated with $150 \mathrm{~ms}$ time windows. The integral of the EMG activity ( Int $_{E M G i}$ ) of TA, GM, RF and BF was evaluated at two epochs relative to $\mathrm{T}_{0}$ : 1) +50 to $+200 \mathrm{~ms}$ (compensatory postural adjustments 1 (CPA1)), and 2) +200 to +350 ms (late compensatory postural adjustments 2 (CPA2)) [4, 24, 27]. The Int $_{E M G i}$ inside each epoch was corrected by subtracting $\operatorname{Int}_{E M G i}$ from -500 to $-450 \mathrm{~ms}$ prior to $\mathrm{T}_{0}$ multiplied by three [24]. As such, positive and negative values indicate increased and decreased muscle activation in relation to the activity recruited before applying the perturbation. The $\operatorname{Int}_{E M G i}$ values were normalised according to the maximum voluntary contraction method $\left(E M G_{\text {norm }}\right)$. Maximal isometric contraction was measured after a warm-up consisting of 3 submaximal isometric contractions for each muscle. To test TA 
and GM muscles the ankle was positioned in neutral position and for the BF and RF muscles the knee was positioned at $90^{\circ}$ [28]. Manual resistance was applied for all muscles [29]. Reciprocal activation and antagonist co-activation were calculated for joint level (muscles that span one joint) and muscle group level (group of muscles that span multiple joints). For the joint level, the muscles acting on the ankle joint (TA/GM pair) and on the knee joint (RF/(GM+BF) pair) were considered. For the muscle group level, the sum of the $E M G_{\text {norm }}$ of all the dorsal (GM and BF) and all the ventral (TA and RF) postural muscles was adopted. Taking into account that the perturbation applied caused a forward oscillation of the subject and the centre of mass position is reestablished through the action of the posterior muscles of the lower limbs and trunk, we assumed the GM and BF muscles as the agonists in postural control response and the TA and RF muscles as their antagonists, respectively.

The antagonist co-activation at joint and muscle group levels during CPA1 and CPA2 were calculated using the following equations [30]:

a) Antagonist co-activation at the joint level:

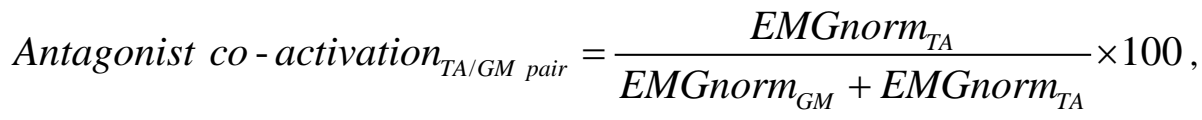

$$
\begin{aligned}
& \text { Antagonist co-activation }_{R F /(B F+G M) \text { pair }}=\frac{E_{\text {aGnorm }}{ }_{R F}}{E M G n o r m_{(B F+G M)}+E M G n o r m_{R F}} \times 100 \text {, }
\end{aligned}
$$

b) Antagonist co-activation at the muscle group level:

$$
\text { Antagonist co-activation }_{\text {ventral/dorsal pair }}=\frac{E M G n o r m_{(T A+R F)}}{E M G n o r m_{(G M+B F)}+E M G n o r m_{(T A+R F)}} \times 100 \text {. }
$$

This approach provides an estimate of the relative activation of the pair of muscles as well as the magnitude of the co-activation. 
The reciprocal activation at joint and muscle group levels during CPA1 and CPA2 was calculated using the following equations [31]:

a) reciprocal activation at the joint level

$$
\text { Reciprocal activation }_{T A / G M \text { pair }}=\text { EMGnorm }_{G M}-\text { EMGnorm }_{T A} \text {, }
$$

Reciprocal activation $_{R F /(B F+G M) \text { pair }}=$ EMGnorm $_{(B F+G M)}-E_{\text {GGnorm }}^{R F}$,

b) Reciprocal activation at the muscle group level

$$
\text { Reciprocal activation }_{\text {ventral/dorsal pair }}=\text { EMGnorm }_{(G M+B F)}-\text { EMGnorm }_{(T A+R F)} \text {. }
$$

The acquired force time series of each trial was used to calculate the CoP fluctuation in the AP direction as:

$$
\operatorname{COP}_{A P}=\frac{M_{x}}{F_{z}},
$$

where $M_{x}$ is the moment in the sagittal plane and $F_{z}$ is the vertical component of the ground reaction force. A fourth-order, zero phase-lag, low-pass Butterworth filter with a cut-off frequency of $20 \mathrm{~Hz}$ [32] was applied to all CoP displacement time series. Only the CoP displacements in the AP direction will be reported, as the perturbations were induced symmetrically. The AP standard deviation $\left(\mathrm{SD}_{\mathrm{AP}}\right)$ and peak-to-peak $\left(\mathrm{P}-\mathrm{P}_{\mathrm{AP}}\right)$ distance of the CoP were measured in the following epochs: (1) +100 to $+250 \mathrm{~ms}$ (CPA1) and (2) +250 to +400 ms (CPA2). These values were selected to compensate the electromechanical delay [33] and were corrected as to base values (obtained during unperturbed standing).

Following an initial evaluation, subjects in the experimental group were given a pair of the unstable shoes and then instructed to wear them as much as possible for at least 8 hours a day, 5 days a week and for 8 weeks, to obtain training effects [6, 9, 12, 
23]. The subjects also received a guide on how to use the shoes. Participants in the control group were told to continue their normal activities and not begin any new exercise regime.

\subsection{Statistics}

The collected data were analysed using the software Statistic Package Social Science (SPSS) from IBM Company (USA). Differences between groups in terms of individual muscle activation, antagonist co-activation and reciprocal activation at joint and muscle group levels, muscle onset and offset and CoP displacement, before and after the 8-week period, were analysed using the Mann-Whitney test. The Friedman ANOVA test was used to compare values obtained in the first and second evaluations in both groups and to compare CPA1 and CPA2 at the different levels in both groups.

\section{Results}

3.1 Influence on muscle activity during CPA at individual, joint and muscle group levels

WUS led to decreased GM activity and increased BF activity when WUS, and to an increased BF activity in the barefoot condition (Figure 1a). No differences were observed between measurements either in the control group (Figure 1c) or between the control group and the experimental group (Table 1). GM activity was higher in CPA1 in all evaluations (Figures 1a and 1c, Table 2).

In Figure 2a, it can be noticed an increase of antagonist co-activation values in CPA1 at TA/GM and ventral/dorsal pairs after WUS only in the unstable shoe condition. In the control group there were no significant differences for these values (Figure 2b). Antagonist co-activation was higher in CPA2 than in CPA1 at TA/GM and ventral/dorsal pairs when WUS for the experimental group (Figure 2a, Table 2), and at all levels in the barefoot condition for the control group (Figure 2b, Table 2). 
The reciprocal activation values decreased at the TA/GM and ventral/dorsal pairs after the 8 weeks of WUS, also only in the unstable shoe condition (Figure 2c) and no differences were observed in the control group (Figure 2d). The experimental group showed higher reciprocal activation values in the first evaluation at ventral/dorsal muscle pair than the control group, while no differences were observed in the second evaluation (Table 1). In both groups, reciprocal activation values were generally higher in CPA1 than in CPA2 (Figures 2c and 2d, Table 2).

\subsection{Influence on CoP displacement during CPA}

In both groups, the $\mathrm{P}-\mathrm{P}_{\mathrm{AP}}$ and $\mathrm{SD}_{\mathrm{AP}}$ decreased in CPA1 in the second evaluation (Figures $1 \mathrm{~b}$ and 1d). However, the experimental group showed higher values of $\mathrm{P}-\mathrm{P}_{\mathrm{AP}}$ and $\mathrm{SD}_{\mathrm{AP}}$ in CPA1 than the control group in the first evaluation, which was not observed after the 8-week period of WUS (Table 1).

\subsection{Influence on muscle latency}

No differences were observed in the experimental group after the 8-week period in TA offset and GM onset. Statistically significant differences between the two groups were only found in TA offset in the barefoot condition in the second evaluation (Figure 3 and Table 1).

\section{Discussion}

\subsection{Influence on muscle activity at the individual muscle level}

The results of this study demonstrate that WUS leads to long-term changes in agonist compensatory postural response. The decrease of GM activity and increase of BF activity after prolonged WUS can express a strategy used by the CNS to minimize energy consumption and/or to optimize postural stability. In fact, a higher activity of the 
larger and more proximal agonist muscles allows these developing compensatory postural response forces with a lower percentage of their maximum capacity, optimising energy consumption [34].

A transfer of postural control synergy for the thigh has been reported as more beneficial to optimise postural stability [35]. The results obtained as to CoP parameters support this finding as they demonstrate higher performance and efficiency of compensatory responses after prolonged WUS. Despite the decreased GM activity, the differences found between CPA1 and CPA2 (Figure 2) and the low values of muscle latency (Figure 3) observed after WUS suggest that the general patterns of postural reactions were preserved regardless of the adaptation mechanisms in terms of muscle activity level.

The transfer of changes associated to WUS to the barefoot condition has not been found in measurements in other functional activities like standing [36] and walking [37]. Our findings suggest that there is a long-term transfer of changes associated with the unstable shoe condition to barefoot condition, in a higher postural control demand task.

4.2 Influence on antagonist co-activation and reciprocal activation at joint and muscle group levels

An increased co-activation at TA/GM pair in CPA1 was observed after WUS for 8 weeks. Previous research has shown that balance training leads to intensification of supraspinal induced pre-synaptic inhibition of Ia afferents $[4,5]$. The interval used to evaluate CPA1 (50-200 ms) included short latency reflexes ( 50 ms), but also long latency reflexes ( 120 ms) [4]. Taking this into account, the increase of antagonist coactivation at TA/GM pair during CPA1 could be explained by an increased pre-synaptic inhibition. It has been hypothesised that some excitability in the segmental circuits of 
the antagonists may allow for their fast recruitment when necessary, such as in the maintenance of equilibrium during postural tasks [38]. Also, the increase of antagonist co-activation could result from the need to reduce the degrees of freedom of body segments. Although in this study kinematic data from body segments were not acquired we can suggest, based on findings from previous gait research [37] and findings obtained from CoP variables, that a decrease of kinematic variability should occur as a result of long-term WUS.

The increase of antagonist co-activation at the TA/GM pair in the experimental group was associated with a decrease of reciprocal activation in the same pair when WUS. This reduction can be associated to the decreased GM activity observed at the individual level since the strength of the disynaptic inhibition is related to the level of motor activity in the agonist [38]. In fact, it was verified that the strength of disynaptic inhibition is reduced during co-contraction of antagonist muscles compared with reciprocal activation [39]. Considering that reciprocal activation is stronger in tasks involving more joint movement [38], the reduction of ankle reciprocal activation obtained in our study could be related to the reduction of $\mathrm{P}-\mathrm{P}_{\mathrm{AP}}$ and $\mathrm{SD}_{\mathrm{AP}}$ after WUS.

The lack of changes in the control group variables between the first and the second evaluation suggests that changes at individual muscle activity, antagonist coactivation and reciprocal activation values in the experimental group were related to WUS.

\section{Conclusion}

The findings obtained indicate that prolonged WUS leads to increased performance and efficiency of postural control adjustments as a result of changes at individual muscle level and at agonist/antagonist muscle relation. These findings, in conjunction with the maintenance of a low muscle latency response encourage the use 
of unstable shoes as a strategy to improve postural control, which assumes particular relevance in rehabilitation.

\section{References}

1. Horak F. Adaptation of automatic postural responses, in The Acquisition of Motor Behavior in Vertebrates, J.e.a. Bloedel, Editor. 1996, MIT Press. p. 57-85.

2. Akram S, Frank J, Patla A and Allum J. Balance control during continuous rotational perturbations of the support surface. Gait and Posture 2008; 27(3): 393-398.

3. Taube W, Gruber M and Gollhofer A. Spinal and supraspinal adaptations associated with balance training and their functional relevance. Acta Physiologica 2008; 193(2): 101-116.

4. Taube W, Gruber M, Beck S, Faist M, Gollhofer A and Schubert M. Cortical and spinal adaptations induced by balance training: correlation between stance stability and corticospinal activation. Acta Physiologica 2007; 189(4): 347-358.

5. Gruber M, Taube W, Gollhofer A, Beck S, Amtage F and Schubert M. Training-specific adaptations of $\mathrm{H}$ - and stretch reflexes in human soleus muscle. Journal of Motor Behaviour 2007; 39(1): 68-78.

6. Ramstrand N, Andersson B and Rusaw D. Effects of an unstable shoe construction on standing balance in children with developmental disabilities: a pilot study. Prosthetics and Orthotics International 2008; 32(4): 422-433.

7. Nigg B, Emery $C$ and Hiemstra L. Unstable shoe construction and reduction of pain in osteoarthritis patients. Medicine and Science in Sports and Exercise 2006; 38(10): 1701-1708.

8. Landry S, Nigg B and Tecante K. Standing in an unstable shoe increases postural sway and muscle activity of selected smaller extrinsic foot muscles. Gait and Posture 2010; 32(2): 215-219.

9. Ramstrand N, Thuesen A, Nielsen D and Rusaw D. Effects of an unstable shoe construction on balance in women aged over 50 years. Clinical Biomechanics 2010; 25(5): 455-460.

10. Turbanski S, Lohrer H, Nauck T and Schmidtbleicher D. Training effects of two different unstable shoe constructions on postural control in static and dynamic testing situations. Physical Therapy in Sport 2011; 12(2): 80-86.

11. Sousa ASP, Tavares JMRS, Macedo R, Rodrigues AM and Santos R. Influence of wearing an unstable shoe on thigh and leg muscle activity and venous response in upright standing. Applied Ergonomics 2012; 43(5): 933-939.

12. Romkes J, Rudmann C and Brunner R. Changes in gait when walking with Masai Barefoot Technique. Clinical Biomechanics 2006; 21(1): 75-81.

13. Boyer $\mathrm{K}$ and Andriacchi $\mathrm{T}$. Changes in running kinematics and kinetics in response to a rockered shoe intervention. Clinical Biomechanics 2009; 24(10): 872-876.

14. Loram I, Maganaris $C$ and Lakie M. Human postural sway results from frequent, ballistic bias impulses by soleus and gastrocnemius. Journal of Physiology 2005; 564(1): 295-311.

15. Fitzpatrick R, Douglas K and McCloskey D. Stable human standing with lower-limb muscle afferents providing the only sensory input. Journal of Physiology 1994; 2536: 395-403.

16. Slijper $\mathrm{H}$ and Latash $\mathrm{M}$. The effects of instability and additional hand support on anticipatory postural adjustments in leg, trunk, and arm muscles during standing. Experimental Brain Research 2000; 135(1): 81-93. 
17. Gelfand I and Latash M. On the problem of adequate language in motor control. Motor Control 1998; 2: 306-313.

18. Horak F and Macpherson J. Postural orientation and equilibrium, in Handbook of physiology, L. Rowell and J. Shepherd, Editors. 1996, Oxford University Press: New York. p. 255-292.

19. Feldman AG. Once more on the equilibrium-point hypothesis (lambda model) for motor control. Journal of Motor Behavior 1986; 18(1): 17-54.

20. Feldman AG and Levin MF. The origin and use of positional frames of reference in motor control. Behavioral Brain Sciences 1995; 18: 723-806.

21. Latash M. Control of Human Movement: Human Kinetics; 1993.

22. Mezzarane R and Kohn A. Control of upright stance over inclined surfaces. Experimental Brain Research 2007; 180(2): 377-388.

23. Nigg B, Hintzen $S$ and Ferber R. Effect of an unstable shoe construction on lower extremity gait characteristics. Clinical Biomechanics 2006; 21(1): 82-88.

24. Santos $\mathrm{M}$, Kanekar $\mathrm{N}$ and Aruin $\mathrm{A}$. The role of anticipatory postural adjustments in compensatory control of posture: 1 . Electromyographic analysis. Journal of Electromyography and Kinesiology 2009; 20(3): 388-397.

25. Di Fabio R. Reliability of computerized surface electromyography for determining the onset of muscle activity. Physical Therapy 1987; 67(1): 43-48.

26. Hodges PW and Bui BH. A comparison of computer-based methods for the determination of onset of muscle contraction using electromyography.

Electroencephalography and Clinical Neurophysiology/Electromyography and Motor Control 1996; 101(6): 511-519.

27. Latash M. Neurophysiological basis of movement. 2nd ed, Champaign: Human Kinetics; 2008.

28. Nishijima Y, Kato T, Yoshizawa M, Miyashita M and lida H. Application of the segment weight dynamic movement method to the normalization of gait EMG amplitude. Journal of Electromyography and Kinesiology 2010; 20(3): 550-557.

29. Netto KJ and Burnett AF. Reliability of normalisation methods for EMG analysis of neck muscles. Work: A Journal of Prevention, Assessment and Rehabilitation 2006; 26(2): 123-130.

30. Kellis E, Arabatzi F and Papadopoulos C. Muscle co-activation around the knee in drop jumping using the co-contraction index. Journal of Electromyography and Kinesiology 2003; 13(3): 229-238.

31. Slijper $\mathrm{H}$ and Latash ML. The effects of muscle vibration on anticipatory postural adjustments. Brain Research 2004; 1015(1-2): 57-72.

32. Winter DA. Biomechanics and Motor Control of Human Movement, New York: Wiley; 1990.

33. Howatson G, Glaister M, Brouner J and van Someren K. The reliability of electromechanical delay and torque during isometric and concentric isokinetic contractions. Journal of Electromyography and Kinesiology 2009; 19(5): 975-979.

34. Chen IH, Kuo KN and Andriacchi TP. The influence of walking speed on mechanical joint power during gait. Gait and Posture 1997; 6(3): 171-176.

35. Runge $C$, Shupert $C$, Horak $F$ and Zajac F. Ankle and hip postural strategies defined by joint torques. Gait and Posture 1999; 10(2): 161-170.

36. Sousa A, Tavares J, Rodrigues A and Santos R. Influence of wearing an unstable shoe on thigh and leg muscle activity and venous response in upright standing. Applied Ergonomics in press 2012.

37. Stöggl T, Haudum A, Birklbauer J, Murrer M and Müller E. Short and long term adaptation of variability during walking using unstable (Mbt) shoes. Clinical Biomechanics 2010; 25(8): 816-822. 
38. Lavoie B, Devanne H and Capaday C. Differential control of reciprocal inhibition during walking versus postural and voluntary motor tasks in humans. Journal of Neurophysiology 1997; 78(1): 429-438.

39. Nielsen J and Kagamihara Y. The regulation of disynaptic reciprocal la inhibition during co-contraction of antagonistic muscles in man. Journal of Physiology 1992; 456: 373391. 


\section{TABLE CAPTIONS}

Table 1: Proof values (p-values) obtained from comparisons made between first (Pre) and second (Post) evaluations in the experimental group (EG) and control group (CG) and between groups. Only significant values are expressed numerically non significant values are represented as ns.

Table 2: Proof values (p-values) obtained from comparisons made between CPA1 and CPA2 in first (Pre) and second (Post) evaluations in the experimental group (EG) and control group (CG). Only significant values are expressed numerically and non significant values are represented as ns. 
TABLES

Table 1: Proof values (p-values) obtained from comparisons made between first (Pre) and second (Post) evaluations in the experimental group (EG) and control group (CG) and between groups. (Only significant values are expressed numerically non significant values are represented as ns.)

\begin{tabular}{|c|c|c|c|c|c|c|c|c|}
\hline Level & Epoch & Variable compared & & $\begin{array}{c}\text { p-value } \\
\text { (Pre vs Post) }\end{array}$ & $\begin{array}{c}\text { p-value } \\
\text { (CG vs EG) }\end{array}$ & & $\begin{array}{c}\text { p-value } \\
\text { (Pre vs Post) }\end{array}$ & $\begin{array}{c}\text { p-value } \\
\text { (CG vs EG) }\end{array}$ \\
\hline \multirow{16}{*}{ 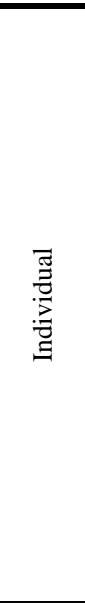 } & \multirow{8}{*}{ CPA1 } & \multirow{2}{*}{$\mathrm{TA}$} & & EG: ns & \multirow{16}{*}{ 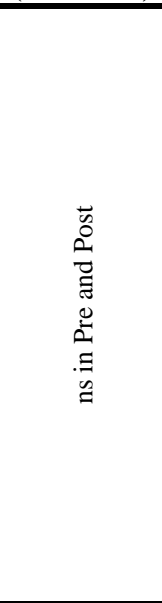 } & & EG: ns & \multirow{16}{*}{ 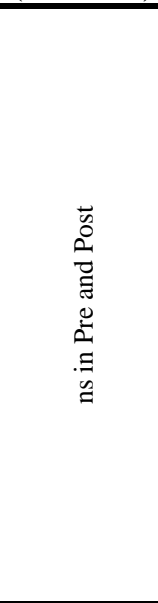 } \\
\hline & & & & CG: ns & & & CG: ns & \\
\hline & & & & EG: $p=0.039$ & & & EG: ns & \\
\hline & & GM & & CG: ns & & & CG: ns & \\
\hline & & $\mathrm{BF}$ & & EG: ns & & & EG: ns & \\
\hline & & $\mathrm{RF}$ & & CG: ns & & & CG: ns & \\
\hline & & $\mathrm{PF}$ & & EG: $p=0.028$ & & & EG: $p=0.023$ & \\
\hline & & $\mathrm{BF}$ & & CG: ns & & & CG: ns & \\
\hline & \multirow{8}{*}{ CPA2 } & \multirow{2}{*}{ TA } & & EG: ns & & & EG: ns & \\
\hline & & & & CG: ns & & & CG: ns & \\
\hline & & \multirow{2}{*}{ GM } & & EG: $p=0.005$ & & & EG: ns & \\
\hline & & & & CG: ns & & & CG: ns & \\
\hline & & \multirow{2}{*}{ RF } & & EG: ns & & & EG: ns & \\
\hline & & & & CG: ns & & & CG: ns & \\
\hline & & \multirow{2}{*}{$\mathrm{BF}$} & & EG: $p=0.006$ & & & EG: $p=0.016$ & \\
\hline & & & & CG: ns & & & CG: ns & \\
\hline \multirow{16}{*}{$\stackrel{. \rightleftarrows}{\circ}$} & \multirow{8}{*}{ CPA1 } & \multirow{2}{*}{$\begin{array}{c}\text { Reciprocal activation } \\
\text { TA/GM pair }\end{array}$} & \multirow{16}{*}{ 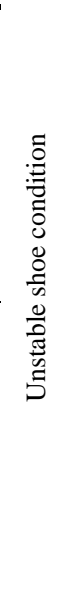 } & EG: $p=0.023$ & \multirow{16}{*}{ 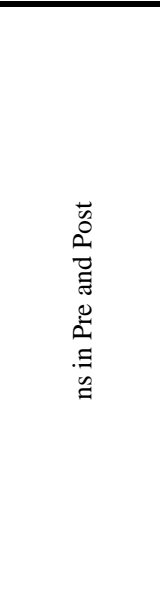 } & & & \\
\hline & & & & CG: ns & & & & \\
\hline & & Reciprocal activation & & EG: ns & & & & \\
\hline & & RF/(BG+GM) pair & & CG: ns & & & & \\
\hline & & Antagonist co-activation & & EG: $p=0.028$ & &. & & \\
\hline & & TA/GM pair & & CG: ns & & 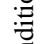 & 0 & $\ddot{\varpi}$ \\
\hline & & Antagonist co-activation & & EG: ns & & రั & שِ & $\stackrel{0}{2}$ \\
\hline & & $\mathrm{RF} /(\mathrm{BF}+\mathrm{GM})$ pair & & CG: ns & & $\ddot{\circ}$ & च & 䒕 \\
\hline & \multirow{8}{*}{ CPA2 } & Reciprocal activation & & EG: $p=0.033$ & & $\stackrel{0}{d}$ & $\underset{I}{\bigcup}$ & 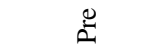 \\
\hline & & TA/GM pair & & CG: ns & & صี & $\Xi$ & $\Xi$ \\
\hline & & Reciprocal activation & & EG: ns & & & $\cong$ & 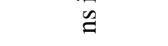 \\
\hline & & RF/(BG+GM) pair & & CG: ns & & & & \\
\hline & & Antagonist co-activation & & EG: ns & & & & \\
\hline & & TA/GM pair & & CG: ns & & & & \\
\hline & & Antagonist co-activation & & EG: ns & & & & \\
\hline & & $\mathrm{RF} /(\mathrm{BF}+\mathrm{GM})$ pair & & CG: ns & & & & \\
\hline \multirow{8}{*}{ 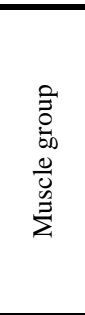 } & \multirow{4}{*}{ CPA1 } & \multirow{2}{*}{$\begin{array}{c}\text { Reciprocal activation } \\
\text { ventral/dorsal pair }\end{array}$} & & EG: $p=0.028$ & Pre: $\mathrm{p}=0.04$ & & \multirow{8}{*}{$\begin{array}{l}\cup \\
\cup \\
\Xi \\
\Xi \\
\cup \\
\Xi \\
\Xi \\
\Xi\end{array}$} & Pre: $\mathrm{p}=0.028$ \\
\hline & & & & CG: ns & Post: ns & & & Post: ns \\
\hline & & Antagonist co-activation & & EG: $p=0.011$ & Pre: ns & & & Pre: ns \\
\hline & & ventral/dorsal pair & & CG: ns & Post: ns & & & Post: ns \\
\hline & & Reciprocal activation & & EG: ns & Pre: $\mathrm{p}=0.003$ & & & Pre: $\mathrm{p}=0.007$ \\
\hline & & ventral/dorsal pair & & CG: ns & Post: ns & & & Post: ns \\
\hline & СРA2 & Antagonist co-activation & & EG: ns & Pre: ns & & & Pre: ns \\
\hline & & ventral/dorsal pair & & CG: ns & Post: ns & & & Post: ns \\
\hline & CPA1 & & & EG: $p=0.001$ & Pre: $\mathrm{p}=0.006$ & & & Pre: $\mathrm{p}=0.004$ \\
\hline ? & CPA2 & $\mathrm{P}-\mathrm{P}_{\mathrm{AP}}$ & & CG: $p=0.033$ & Post: ns & & U & Post: ns \\
\hline$\dot{0}$ & CPA1 & & & EG: $p=0.023$ & Pre: $\mathrm{p}=0.01$ & &.$\Xi$ & Pre: $\mathrm{p}=0.005$ \\
\hline & CPA2 & $\mathrm{SD}_{\mathrm{AP}}$ & & CG: $p=0.033$ & Post: ns & & & Post: ns \\
\hline & & TA offset & & & & & & Pre: ns \\
\hline 岂 & & TA offset & & $\Xi$ ฮ & $\Xi \Xi \tilde{\sigma}$ & & $\Xi$ ฮี & Post: $\mathrm{p}=0.003$ \\
\hline$\sum_{\Sigma}^{\Xi}$ & & GM onset & & $\cong \bigcup_{\text {III }} \cup$ & $\approx \cong$ & & 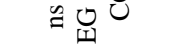 & Pre: ns \\
\hline & & & & & & & & Post: ns \\
\hline
\end{tabular}


Table 2: Proof values (p-values) obtained from comparisons made between CPA1 and CPA2 in first (Pre) and second (Post) evaluations in the experimental group (EG) and control group (CG). (Only significant values are expressed numerically and non significant values are represented as ns.)

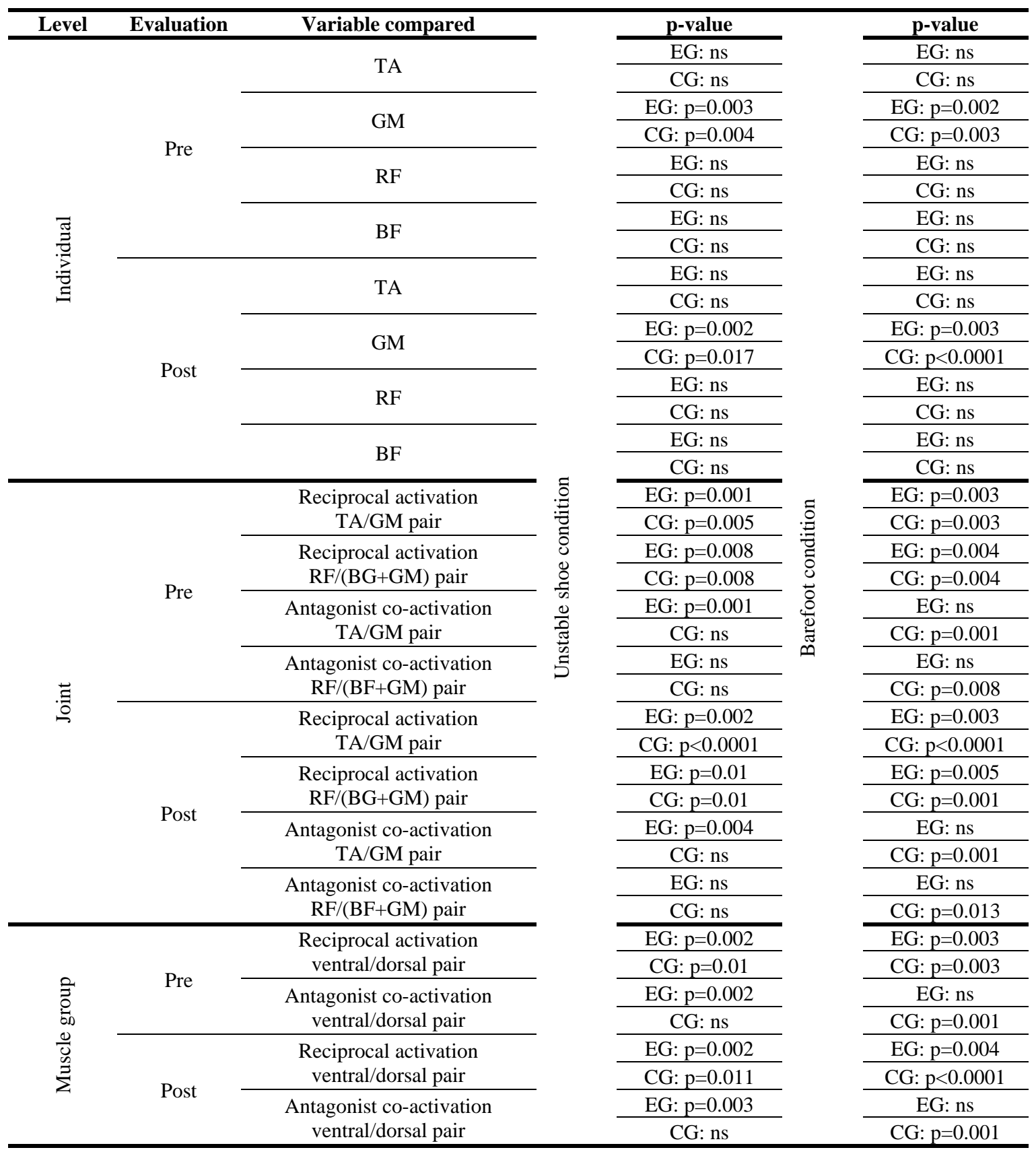




\section{FIGURE CAPTIONS}

Figure 1: Representation of mean (bars) and SD (error bars) values of GM, TA, BF and RF EMG activity (a) and peak to peak and standard deviation values of CoP displacement in the $\mathrm{AP}$ direction $\left(\mathrm{P}-\mathrm{P}_{\mathrm{AP}}, \mathrm{SD}_{\mathrm{AP}}\right)$ (b) during $\mathrm{CPA}$, in barefoot and unstable shoe conditions, before (Pre) and after (Post) 8 weeks of WUS by the experimental group (a) and (b) and before and after the same period by the control group (c) and (d). $\left({ }^{*} \mathrm{p}<0.05,{ }^{* *} \mathrm{p}<0.01\right)$

Figure 2: Antagonist co-activation (a) and reciprocal activation (c) values at joint and muscle group (MG) levels obtained during CPA before (Pre) and after (Post) 8 weeks of WUS by the experimental group and before and after the same period by the control group (b) and (d). $\left({ }^{*} \mathrm{p}<0.05,{ }^{* *} \mathrm{p}<0.01\right)$

Figure 3: Onset and offset latency of leg muscles to an external perturbation before (Pre) and after (Post) 8 weeks of WUS by the experimental group and before and after the same period by the control group, in barefoot and unstable shoe conditions. The muscle latency was only evaluated in TA and GM, as the main changes in muscle activity level, antagonist co-activation and reciprocal activation occurred at this level. $\left({ }^{* *} \mathrm{p}<0.01\right)$ 

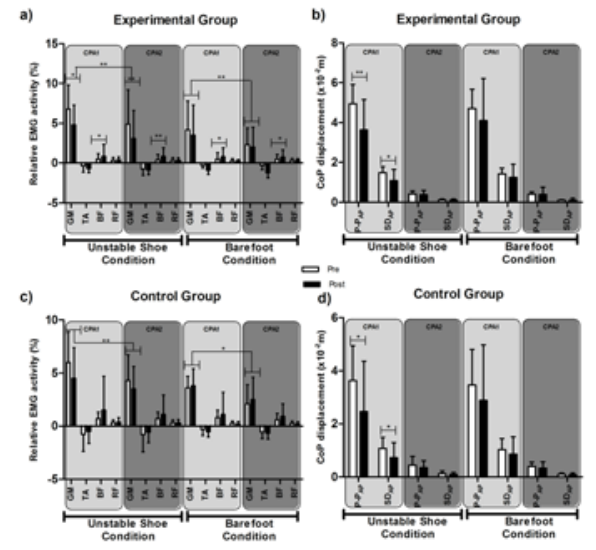

Figure 1
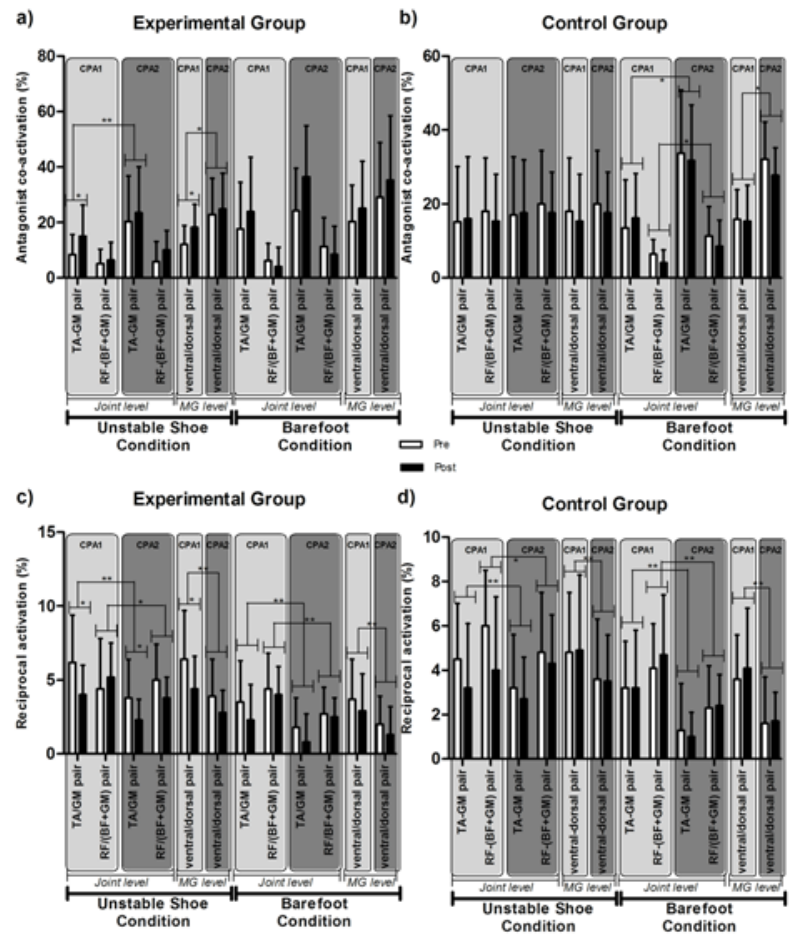

Figure 2 


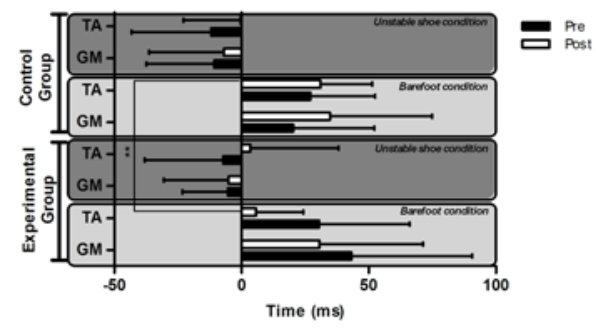

Figure 3 